\title{
474641 - TRANEXAMIC ACID AND TRANSFUSION IN ORTHOTOPIC LIVER TRANSPLANTATION
}

\author{
Simon Gower, MD, Coimbatore Srinivas, MD, Reza Ghaffari, Anesthesiologist, \\ Nicholas Mitsakakis, M.Sc., Stuart McCluskey, MD \\ Anesthesia, Toronto General Hospital, Toronto, ON, Canada
}

Introduction: Orthotopic liver transplantation (OLT) is associated with significant blood loss and massive transfusion (> 5 units packed red blood cells pRBCs). Antifibrinolytic agents have been shown to reduce blood transfusion in randomized controlled trials1. However, the effectiveness and safety of these agents in clinical practice has not been demonstrated. The objective of this study was to determine if the rates of blood transfusion and massive transfusion were lower in patients undergoing OLT who received tranexamic acid (TA).

Methods: Following Research Ethics Board approval, propensity score methods were used to match 177 patients who received TA to 177 similar patients who did not receive any antifibrinolytic from a pool 695 consecutive patients who received OLT between January, 1999 and December 2006. The effects of TA on blood transfusion and massive transfusion were compared in the matched groups using the Wilcox test for nonparametric continuous variables and conditional logistic regression for categorical variables.

Results: The two matched groups were well balanced in terms of all measured preoperative variables: age, height, weight, gender, etiology of liver failure, co-morbidities, and preoperative predictors of massive transfusion (hemoglobin concentration, INR, platelet count, albumin concentration and creatinine). The median number of PRBC units transfused in those given TA was significantly lower (median 4 [IQR: 1, 7] than those receiving no antifibrinolytic medication (median 5 [IQR: 2, 9] $(\mathrm{p}=0.019)$. In patients administered TA there was a significant increase in the proportion of those receiving no blood transfusion and 1-2 unit transfusions and a significant reduction in the incidence of massive transfusion (> 5 units of RBC) compared to those receiving no TA.

Discussion: TA is associated with a reduction blood transfusion requirement in OLT. Whether this improves patient safety and outcome needs to be determined.

References: 1. Am J Transplant 2007; 7:185-94.

\begin{tabular}{|c|c|c|c|c|}
\hline & PRBC $=0$ units $(\%)$ & PRBC $=1-2$ units $(\%)$ & PRBC $=3-5$ units $(\%)$ & PRBC $>5$ units $(\%)$ \\
\hline No TA & 13 & 14.1 & 24.3 & 48.6 \\
\hline TA & 21.5 & 18.1 & 22.5 & 37.9 \\
\hline P value & 0.039 & 0.025 & NS & 0.046 \\
\hline
\end{tabular}

